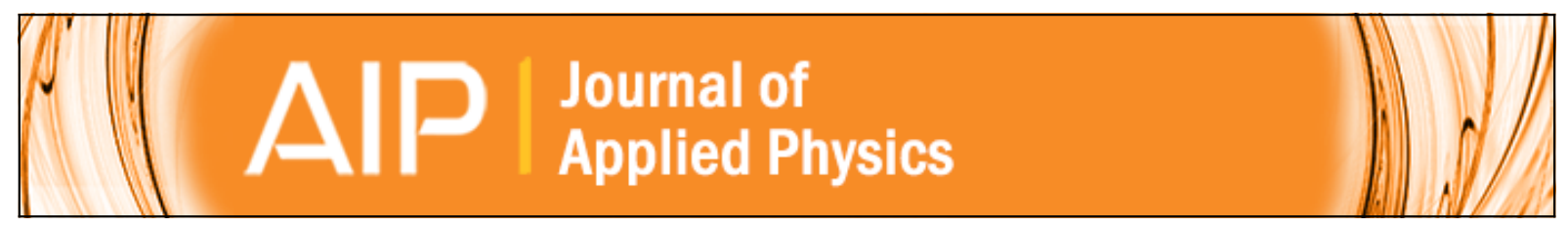

\title{
Transport in random quantum dot superlattices
}

I. Gómez, F. Domínguez-Adame, E. Diez, and P. Orellana

Citation: Journal of Applied Physics 92, 4486 (2002); doi: 10.1063/1.1503393

View online: http://dx.doi.org/10.1063/1.1503393

View Table of Contents: http://scitation.aip.org/content/aip/journal/jap/92/8?ver=pdfcov

Published by the AIP Publishing

\section{Articles you may be interested in}

Internal quantum efficiency of III-nitride quantum dot superlattices grown by plasma-assisted molecular-beam epitaxy

J. Appl. Phys. 109, 103501 (2011); 10.1063/1.3590151

Functionalizing self-assembled GaN quantum dot superlattices by Eu-implantation J. Appl. Phys. 108, 084306 (2010); 10.1063/1.3496624

Time-resolved photoluminescence of type-II quantum dots and isoelectronic centers in $\mathrm{Zn}-\mathrm{Se}-\mathrm{Te}$ superlattice structures

Appl. Phys. Lett. 92, 032106 (2008); 10.1063/1.2835699

GaN quantum dot superlattices grown by molecular beam epitaxy at high temperature

J. Appl. Phys. 102, 073517 (2007); 10.1063/1.2787155

Temperature dependence of photoluminescence spectra in InAs/GaAs quantum dot superlattices with large thicknesses

J. Appl. Phys. 82, 4489 (1997); 10.1063/1.366255

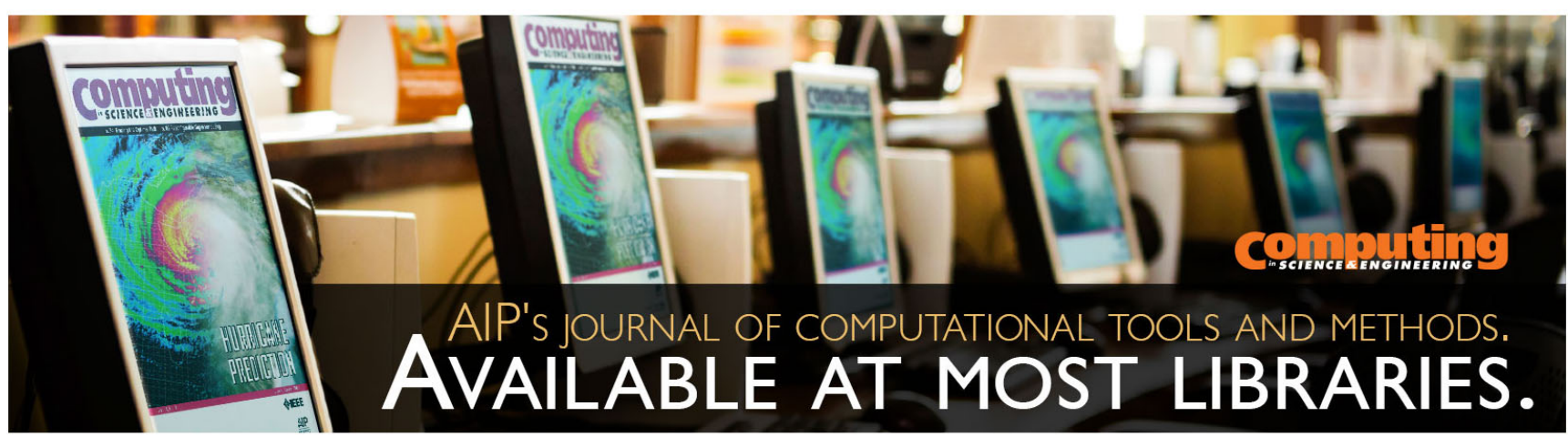




\title{
Transport in random quantum dot superlattices
}

\author{
I. Gómez ${ }^{\text {a) }}$ F. Domínguez-Adame, and E. Diez \\ GISC, Departamento de Física de Materiales, Universidad Complutense, E-28040 Madrid, Spain \\ P. Orellana \\ Departamento de Física, Universidad Católica del Norte, Casilla 1280, Antofagasta, Chile
}

(Received 12 February 2002; accepted for publication 2 July 2002)

\begin{abstract}
We present a model based on the two-dimensional transfer matrix formalism to calculate single-electron states in a random wide-gap semiconductor quantum dot superlattice. With a simple disorder model both the random arrangement of quantum dots (configurational disorder) and the spatial inhomogeneities of their shape (morphological disorder) are considered. The model correctly describes channel mixing and broadening of allowed energy bands due to elastic electron scattering by disorder. (C) 2002 American Institute of Physics. [DOI: 10.1063/1.1503393]
\end{abstract}

\section{INTRODUCTION}

The latest advances in nanotechnology make it possible to grow quantum dot (QD) superlattices. ${ }^{1,2}$ In view of the analogy between atoms and QDs, it is expected that strongly confined levels overlap when QDs are closely packed. Although this analogy cannot be complete since carriers in QDs are influenced by phonons, defects, and interface states, the resulting collective states will depend on the arrangement of QDs. In this sense, QD arrays grown by molecular beam epitaxy can be completely random, ${ }^{3,4}$ partially regimented ${ }^{1,2}$ or may be regularly stacked (high regimentation). ${ }^{5}$ Electronic states in highly regimented QDs are adequately described within the envelope-function approximation by the three dimensional Kronig-Penney model. The occurrence of minibands has been established. ${ }^{6}$ However, the lack of periodicity in random QDs superlattices requires different approaches.

In the present work we present a two-dimensional effective-mass model to study the influence of scattering by disorder on electron transport through random QD superlattices. We use the discretized Ben Daniel-Duke equation, and boundary conditions are discussed. The scattering solutions are calculated by means of the transfer-matrix method for an arbitrary QD superlattice. The model is worked out in a twodimensional space for computational limitations, although it will be clear that generalization to three dimensions is rather straightforward. Finally, we present the numerical results for the conductance of random arrays of coupled QDs and the main conclusions of the work.

\section{MODEL}

We consider the Daniel-Duke equation for the electron envelope function $\chi(y, z)$ with a constant effective mass $m^{*}$ at the $\Gamma$ valley. In order to calculate the single electron states, the whole sample is divided into three regions. Regions I and III are the contacts, while region II contains the array left (I) and right (III) contacts and the random QDs (II). Electron scattering by disorder takes place in the region III. Figure 1

${ }^{a)}$ Electronic mail: igcuesta@valbuena.fis.ucm.es shows a schematic view of the three spatial regions. Using the Daniel-Duke equation and a mesh with lattice spacings $a_{y}$ and $a_{z}$ in the $Y$ and $Z$ directions, and defining $t_{y}$ $\equiv-\hbar^{2} /\left(2 m^{*} a_{y}^{2}\right)$ and $t_{z} \equiv-\hbar^{2} /\left(2 m^{*} a_{z}^{2}\right)$, we obtain the following discrete effective-mass equation:

$$
\begin{gathered}
t_{z}\left(\chi_{n+1, m}+\chi_{n-1, m}\right)+t_{y}\left(\chi_{n, m+1}+\chi_{n, m-1}\right) \\
+\left(U_{n, m}-2 t_{z}-2 t_{y}\right) \chi_{n, m}=E \chi_{n, m} .
\end{gathered}
$$

The potential term $U_{n, m}$ in Eq. (1) is given by the conduction-band edge energy at the point $\left(n a_{y}, m a_{z}\right)$. Therefore, disorder enters the equation via this diagonal term. Contacts are characterized by flatband conditions, $U_{n, m}=0$, in the absence of applied electric field. The effects of the applied field can easily be taken into account within the present approach by adding a linearly varying potential of the form $U_{n, m}(F)=-e F m a_{z}$ in the region II, $F$ being the applied field.

\section{TRANSFER MATRIX FORMALISM}

In order to solve the tight-binding Eq. (1) we use the transfer matrix method based on the solutions calculated for each slice of the system along the $Z$ direction. ${ }^{7}$ For the sake of simplicity, we define $\boldsymbol{\phi}_{n}(n=0,1, \ldots, N+1)$ as a vector with the components $\phi_{n}^{(m)} \equiv \chi_{n, m}(m=1, \ldots, M)$. Here $M$ and $N+1$ are the number of mesh divisions in the $Y$ and $Z$ directions, respectively. Thus, Eq. (1) can be rewritten in a more compact form

$$
\left(\begin{array}{c}
\boldsymbol{\phi}_{n-1} \\
\boldsymbol{\phi}_{n}
\end{array}\right)=\left(\begin{array}{cc}
t_{z}^{-1}\left(E \mathcal{I}-\mathcal{M}_{n}\right) & -\mathcal{I} \\
\mathcal{I} & \mathcal{O}
\end{array}\right)\left(\begin{array}{c}
\boldsymbol{\phi}_{n} \\
\boldsymbol{\phi}_{n+1}
\end{array}\right)
$$

where $\mathcal{I}$ and $\mathcal{O}$ are the $M \times M$ identity and null matrices, respectively. The matrix $\mathcal{M}_{n}$ splits into the form $\mathcal{M}_{n}=\mathcal{R}_{n}$ $+\mathcal{B}_{n}$. The diagonal elements of the symmetric tridiagonal matrix $\mathcal{R}_{n}$ are $\left(\mathcal{R}_{n}\right)_{m m}=U_{n, m}-2 t_{z}-2 t_{y}$ while off-diagonal elements equal $t_{y}$. The matrix $\mathcal{B}_{n}$ depends on the boundary conditions to be specified later.

We can obtain the expression for the envelope function amplitudes in the left contact as a function of the amplitudes in the right one 
Self-assembled quantum dots

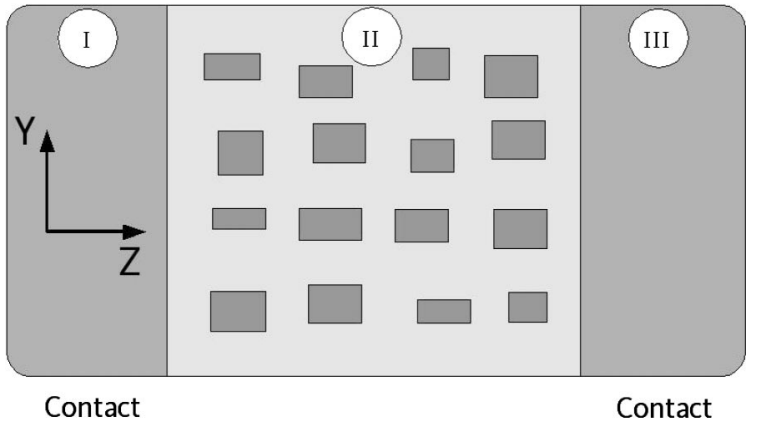

FIG. 1. Schematic view of the sample. Regions I and III are the electrical leads of the sample (contacts) and electrons undergo scattering processes only at region II.

$$
\begin{aligned}
\left(\begin{array}{c}
\boldsymbol{\phi}_{0} \\
\boldsymbol{\phi}_{1}
\end{array}\right) & =\mathcal{T}^{(N)}\left(\begin{array}{c}
\boldsymbol{\phi}_{N} \\
\boldsymbol{\phi}_{N+1}
\end{array}\right), \\
\mathcal{T}^{(N)} & \equiv \prod_{n=1}^{N}\left[\begin{array}{cc}
t_{z}^{-1}\left(E \mathcal{I}-\mathcal{M}_{n}\right) & -\mathcal{I} \\
\mathcal{I} & \mathcal{O}
\end{array}\right],
\end{aligned}
$$

where $\mathcal{T}^{(N)}$ is the transfer matrix for the heterostructure.

\section{SCATTERING SOLUTIONS}

The envelope functions within the contacts are determined by the boundary conditions. We use open boundary conditions in the $Z$ direction and periodic on each slice in the $Y$ direction. Consequently, all elements of $\mathcal{R}_{n}$ vanish except $\left(\mathcal{R}_{n}\right)_{1 M}=\left(\mathcal{R}_{n}\right)_{M 1}=t_{y}$. The open boundary condition results in plane wave solutions in the $Z$ direction, while the periodic boundary condition yields an energy quantization in the $Y$ direction. This quantization results in a number of transverse channels equal to the number of points in the transverse mesh direction. We assume perfect leads so that $U_{n, m}$ is a constant within the contacts. In this case the voltage only drops across the region II. Applying the periodic boundary condition $\chi_{n, 1}=\chi_{n, M+1}$, a particular solution of Eq. (1) is given by

$$
\begin{aligned}
\chi_{n, m}^{l}= & \frac{1}{\sqrt{\mathcal{N}_{l}}} \exp \left(i \frac{2 \pi l}{M} m\right) \exp \left(i k_{l} a_{z} n\right) \\
& +\sum_{j=1}^{M} \hat{r}_{l j} \frac{1}{\sqrt{\mathcal{N}_{j}}} \exp \left(i \frac{2 \pi j}{M} m\right) \\
& \times \exp \left(-i k_{j} a_{z} n\right), \quad(m, n) \in \mathrm{I},
\end{aligned}
$$

at the left contact while at the right contact we have

$$
\begin{aligned}
\chi_{n, m}^{l}= & \sum_{j=1}^{M} \hat{t}_{l j} \frac{1}{\sqrt{\mathcal{N}_{j}}} \exp \left(i \frac{2 \pi j}{M} m\right) \exp \left(i k_{j} a_{z} n\right), \\
& (m, n) \in \mathrm{III},
\end{aligned}
$$

where the normalization constant is chosen to ensure that all the propagating modes carry the same current

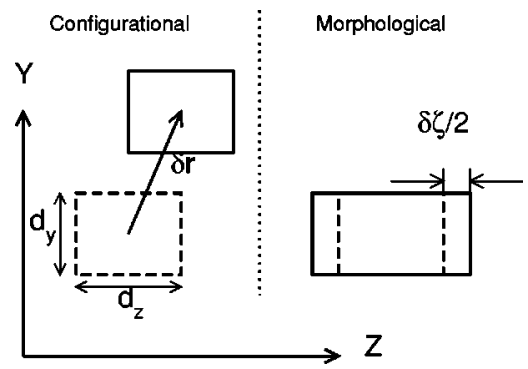

FIG. 2. Schematic view of configurational and morphological disorder. In the former case the QD shift its position and amount $\delta \mathbf{r}$ while its nominal size $d_{y} \times d_{z}$ remains unchanged. In the latter case, the center of the QD does not change while its size along the $Z$ axis increases or decreases an amount $\delta \zeta$.

$$
\mathcal{N}_{j}=\frac{1}{a_{y}^{2}} \sin ^{2}\left(\frac{2 \pi j}{M}\right)+\frac{1}{a_{z}^{2}} \sin ^{2}\left(k_{j} a_{z}\right)
$$

and

$$
k_{j}=\frac{1}{a_{z}} \cos ^{-1}\left\{\frac{1}{2 t_{z}}\left[E-2 t_{y}\left(\cos \frac{2 \pi j}{M}-1\right)\right]+1\right\} .
$$

These expressions remain valid for an applied electric field $F$ provided $E$ is replaced by $E+e V$ in Eq. (4d), where $V$ $=F L$ is the applied voltage and $L$ is the length of region II.

The matrices $\hat{r}$ and $\hat{t}$ in Eqs. (4) are the reflection and transmission matrices and they account for the channel mixing due to scattering. Thus, $\hat{r}_{i j}$ represents the probability amplitude for an electron in channel $i$ to be reflected into the channel $j$. Note that the solution within region II is not required to obtain $\hat{r}$ and $\hat{t}$. All scattering properties of the system are described by the $\hat{t}$ and $\hat{r}$ which mix the different channels. In particular, we can calculate the conductance. From the Landauer-Büttiker formalism, ${ }^{8}$ the zero temperature two-leads multichannel conductance can be calculated using de Fisher-Lee formula ${ }^{9}$

$$
G=\frac{2 e^{2}}{h} \operatorname{Tr}\left(\hat{t}^{\dagger} \hat{t}\right),
$$

where $\operatorname{Tr}$ stands for the trace of the matrix.

\section{CONFIGURATIONAL AND MORPHOLOGICAL DISORDER}

In order to describe epitaxially random QD superlattices, we consider they are arranged on a nonregular lattice (configurational disorder). There also exists another type of disorder that is due to inhomogeneities of QD shapes (morphological disorder). To simulate both types of disorder we consider a superlattice of rectangular QDs. Sizes of QDs and their displacements from the regular lattice sites are taken to be random (see Fig. 2). For the sake of simplicity, we assume that the confining band offset $\Delta E_{c}$ is the same for every QD. This is not a serious shortcoming since spatial inhomogeneities of the conduction-band offset and fluctuations of QDs shapes yield essentially the same results. In addition, our model could easily deal with inhomogeneous $\Delta E_{c}$ if further improvements are required. 


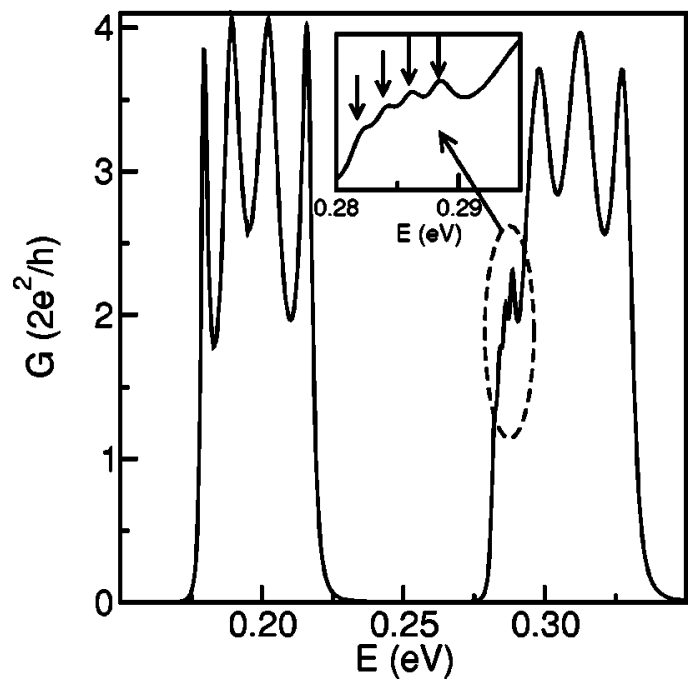

FIG. 3. Conductance vs energy for a two-dimensional ordered superlattice of $4 \times 4$ QDs made of $\operatorname{In}_{x} \mathrm{Ga}_{1-x}$ As in GaAs (solid line) at zero bias. The inset shows an enlarged view of the lower conductance peak of the second miniband.

For the sake of clarity we consider the effects of configurational and morphological disorder separately. Configurational disorder means that each $\mathrm{QD}$ is shifted from its regular position by $\delta \mathbf{r}=(\delta y, \delta z)$ while its size $d_{y} \times d_{z}$ remains unchanged (see left panel of Fig. 2). Here $\delta y$ and $\delta z$ are random uncorrelated variables with zero mean and distributed according to box probability functions of width $W_{y}$ and $W_{z}$, respectively. To simulate the change of the shape (morphological disorder) we change the size of a QD in the $Z$ direction an amount $\delta \zeta$ while its center stays on a regular lattice (see right panel of Fig. 2). Here $\delta \zeta$ is a uniformly distributed variable with zero mean and width $W_{\zeta}$.

\section{RESULTS}

We have performed several numerical calculations in order to study the influence of both configurational and morphological disorder over the conductance of random QDs superlattices made of $\mathrm{GaAs}-\mathrm{In}_{x} \mathrm{Ga}_{1-x}$ As heterojunctions. To elucidate the effects of randomness, we have considered regimented and disordered $4 \times 4$ QD superlattices. The conduction band offset, $\Delta E_{c}$, is taken to be $70 \%$ of the difference of the gaps $\Delta E_{g}$ in a strained GaAs- $\mathrm{In}_{x} \mathrm{Ga}_{1-x}$ As heterojunction, where $\Delta E_{g}=1.45 x \mathrm{eV}$. For definiteness we set $x=0.35$ and consequently $\Delta E_{c}=0.35 \mathrm{eV}$. In addition, since we are mainly interested in the effects of the coupling between the QDs via the high band gap semiconductor rather in the confined levels of individual QD, we have taken $m^{*}$ $=0.067$ in units of the free electron mass, corresponding to the embedding semiconductor. Let us mention that the model can be easily generalized to include a different effective mass inside the QDs. The size of the regimented QDs was taken to be $d_{y} \times d_{z}$ with $d_{y}=8.0 \mathrm{~nm}$ and $d_{z}=1.6 \mathrm{~nm}$. The separation between centers in the regimented superlattice is 14.0 and $6.8 \mathrm{~nm}$ along the $Y$ and $Z$ axes, respectively. The number of mesh points along the two spatial directions are $M=50$ and $N+1=39$.

As typical results of our simulations, Fig. 3 shows the

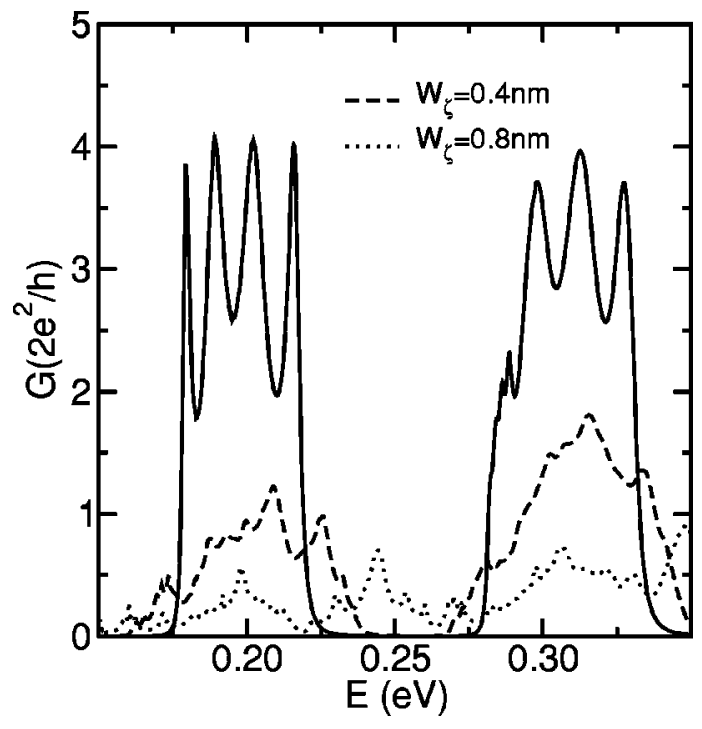

FIG. 4. Conductance vs energy for a two-dimensional superlattice of $4 \times 4$ QDs with morphological disorder: $W_{\zeta}=0.4 \mathrm{~nm}$ (dashed line) and $W_{\zeta}=0.8$ $\mathrm{nm}$ (dotted line). Results are compared to the conductance of the ordered superlattice shown in Fig. 3 (solid line).

conductance versus energy measured from the conduction band edge in $\mathrm{In}_{x} \mathrm{Ga}_{1-x} \mathrm{As}$, in the absence of the electric field. The coupling between QDs splits the energy levels and results in the formation of minibands. ${ }^{6}$ From Fig. 3 we observe the occurrence of two well-defined minibands below the barrier when disorder is absent, in agreement with previous results. ${ }^{6}$ Each band is characterized by four main conductance peaks and each peak is the convolution of four closer peaks that cannot be resolved except in the low energy region of the higher miniband (see inset of Fig. 3). These four peaks merge into a single broader one due to the large spacing between QDs along the $Y$ direction. Thus, the coupling between neighbor QDs is weaker along this direction $\left(\mid t_{y}\right.$ $|<| t_{z} \mid$ ), finally resulting in a smaller splitting of the QD levels. This merging effect is smaller in the higher miniband (see inset of Fig. 3) because of a higher overlap of the envelope functions, which manifests itself via the broadening of the four main peaks.

Transport through the miniband changes as soon as some degree of randomness is considered in the model. Mean size and separation between QDs as well as their fluctuations values strongly depend on the growth conditions (e.g., growth temperature) and subsequent thermal treatments. ${ }^{4}$ As an example, Fig. 4 shows the conductance for two different degrees of morphological disorder, $W_{\zeta}=0.4 \mathrm{~nm}$ (dashed line) and $W_{\zeta}=0.8 \mathrm{~nm}$ (dotted line). In addition, Fig. 5 displays the conductance for two different degrees of configurational disorder, $W_{y}=2.0 \mathrm{~nm}$ and $W_{z}=1.2 \mathrm{~nm}$ (dotted line), and $W_{y}$ $=2.0 \mathrm{~nm}$ and $W_{z}=0 \mathrm{~nm}$ (dashed line). All the curves for the disordered samples were obtained by averaging over 100 realizations of the disorder. In both figures the solid line corresponds to the regimented QD superlattice shown in Fig. 3.

As the main point, we notice that electronic states in a random QD superlattice behave like those in amorphous materials, in the sense that with increase in disorder the conductance strongly decreases while the allowed energies broaden 


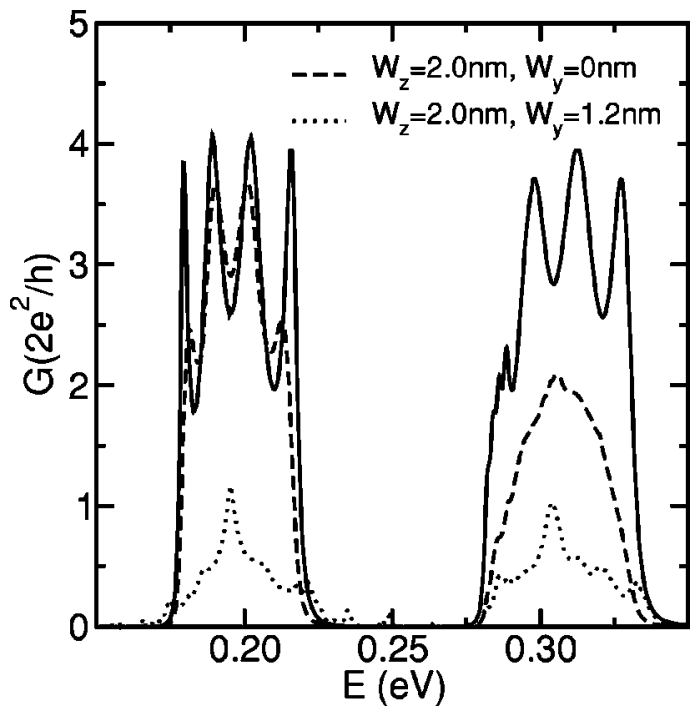

FIG. 5. Conductance vs energy for a two-dimensional superlattice of $4 \times 4$ QDs with configurational disorder: $W_{y}=2.0 \mathrm{~nm}$ and $W_{z}=1.2 \mathrm{~nm}$ (dotted line), and $W_{y}=2.0 \mathrm{~nm}$ and $W_{z}=0 \mathrm{~nm}$ (dashed lines). Results are compared to the conductance of the ordered superlattice shown in Fig. 3 (solid line).

due to the fluctuations of QD energy levels. It is clear from Fig. 4 that the gap disappears in the case of strong morphological disorder as resonant tunneling is suppressed due to large energy spacing between levels of different QDs. However, the effects of configurational disorder on the band structure are less profound, in the sense that broadening is negligible for realistic values of the parameters, although the decrease of the conductance is still observed (see Fig. 5). Notice that the conductance reaches its maximum at the energy of the individual QD quasilevels, as expected.

Concerning the effects of a uniform applied electric field, we have computed the conductance for a given Fermi energy as a function of the applied bias $V$. Two cases, $E_{f}$ $=0.17 \mathrm{eV}$ and $E_{f}=0.27 \mathrm{eV}$, corresponding to the bottom of the two minibands, has been considered. The reason for this choice is to observe the behavior of the conductance when the miniband goes down and crosses the Fermi level as the applied voltage increases. Figure 6 shows the conductance versus applied bias for regimented as well as random QD superlattices in the two cases, where voltage was assumed to drop linearly across region II. Regimented QD superlattices present three well-defined negative $d G / d V$ regions due to resonant tunneling through the QDs. The observed peak-tovalley ratios are worse for disordered samples.

\section{CONCLUSIONS}

In summary, we have presented a method to study electron transport through random QD superlattices. The method is based on the transfer matrix formalism applied to the discrete Ben Daniel-Duke Hamiltonian for the electron envelope function. A careful analysis of the scattering solutions under appropriate boundary conditions (periodic in the lateral direction and open along the longitudinal one) allows us to obtain the two-channel conductance. For regimented QDs

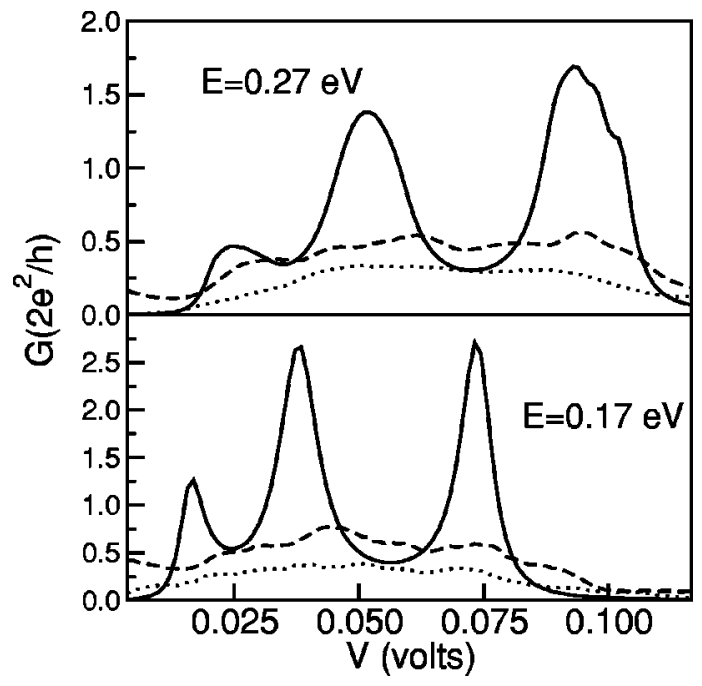

FIG. 6. Conductance vs applied bias for a two-dimensional ordered superlattice of $4 \times 4$ QDs, made of $\operatorname{In}_{x} \mathrm{Ga}_{1-x} \mathrm{As}$ in GaAs (solid line), when the incoming electron energy is $E_{f}=0.17 \mathrm{eV}$ (lower panel) and $E_{f}=0.27 \mathrm{eV}$ (upper panel). Results are compared to random $4 \times 4$ superlattices with configurational disorder for $W_{y}=2.0 \mathrm{~nm}$ and $W_{z}=1.2 \mathrm{~nm}$ (dotted line) or with morphological disorder with $W_{\zeta}=0.8 \mathrm{~nm}$ (dashed line).

(regular QD superlattices) the conductance shows clear signatures of the miniband structure, as previously predicted by a Kronig-Penney model for strongly coupled QDs. ${ }^{6}$ However, the novelty of the model is that it allows for description of random QD superlattices. Two models of disorder (configurational and morphological) have been studied. Disorder reduces the conductance due to Anderson localization of the envelope functions and broadens the allowed energy bands. This broadening is negligible for configurational disorder. The characteristic $G-V$ presents several regions of negative $d G / d V$, although the peak-to-valley ratios strongly decrease with disorder.

\section{ACKNOWLEDGMENTS}

Work in Madrid was supported by DGI-MCyT (Project No. MAT2000-0734) and CAM (Project No. 07N/0075/ 2001). P. Orellana would like to thank Milenio ICM P99135-F and Cátedra Presidencial de Ciencias for financial support. The authors would like to thank Andrei Malishev for comments on the manuscript.

${ }^{1}$ J. L. Liu, W. G. Wu, A. Balandin, G. L. Jin, and K. L. Wang, Appl. Phys. Lett. 74, 185 (1999)

${ }^{2}$ J. L. Liu, W. G. Wu, A. Balandin, G. L. Jin, Y. H. Luo, S. G. Thomas, and K. L. Wang, Appl. Phys. Lett. 75, 1745 (1999).

${ }^{3}$ I. Tanaka, I. Kamiya, H. Sasaki, N. Qureshi, S. J. Allen, and P. M. Petroff, Appl. Phys. Lett. 74, 844 (1999).

${ }^{4}$ P. C. Sharma, K. W. Alt, D. Y. Yeh, and K. L. Wang, Appl. Phys. Lett. 75, 1273 (1999).

${ }^{5}$ G. Springholz, V. Holy, M. Pinczolits, and G. Bauer, Science 282, 734 (1998).

${ }^{6}$ O. L. Lazarenkova and A. A. Balandin, J. Appl. Phys. 89, 5509 (2001).

${ }^{7}$ Computational Physics, edited by K. H. Hoffmann and M. Scheiber (Springer, Berlin, 1996).

${ }^{8}$ R. Landauer, IBM J. Res. Dev. 1, 223 (1957); M. Büttiker, Phys. Rev. Lett. 57, 1761 (1986); IBM J. Res. Dev. 32, 63 (1988); 32, 317 (1988).

${ }^{9}$ D. Fisher and P. A. Lee, Phys. Rev. B 23, 6851 (1981). 